\title{
Desenvolvimento de aplicativo de orientações domiciliares para indivíduos com encefalopatia crônica não progressiva da infância
}

\author{
Developing household guidelines for individuals with \\ nonprogressive chronic childhood encephalopathy
}

\author{
Amanda Caroline Lobato Dias ${ }^{1}$, Cássia Oliveira Cabral da Paz², Isabelle Farias Gomes ${ }^{3}$, Carina Alves \\ Costa $^{4}$, Janine Brasil de Araújo Moraes ${ }^{5}$, Izidio Sousa de Carvalho ${ }^{6}$, Larissa Salgado de Oliveira Rocha ${ }^{7}$
}

\begin{abstract}
'Autora para correspondência. Universidade do Estado do Pará. Belém, Pará, Brasil. ORCID: 0000-0003-2940-2057. amanda_cld97@yahoo.com.br 2Universidade do Estado do Pará. Belém, Pará, Brasil. ORCID: 0000-0002-4959-2452. cassiapaz7@gmail.com 3universidade do Estado do Pará. Belém, Pará, Brasil. ORCID: 0000-0002-0485-0315. isabellefisio@outlook.com 4Universidade do Estado do Pará. Belém, Pará, Brasil. ORCID: 0000-0002-2331-8121. costaalvescarina@gmail.com 5Universidade do Estado do Pará. Belém, Pará, Brasil. ORCID: 0000-0003-0575-7179. jani.brasil.jb@gmail.com שUniversidade Federal do Pará. Belém, Pará, Brasil. ORCID: 0000-0002-1994-2693. izidiocarvalho88@gmail.com 7Universidade do Estado do Pará. Belém, Pará, Brasil. ORCID: 0000-0002-6919-4160. lari1980@gmail.com
\end{abstract}

RESUMO | INTRODUÇÃo: A Encefalopatia Crônica Não Progressiva da Infância (ECNPI) é uma condição de deficiência física não progressiva decorrente de uma lesão no sistema nervoso central em desenvolvimento. Ela apresenta desordens motoras, no tônus muscular e na postura, com isso provocando uma deficiência na coordenação da ação muscular e dos movimentos, incapacidade ou dificuldade na manutenção postural. Com o crescimento e desenvolvimento das crianças pode ocorrer um agravo do quadro de saúde, como aparecimento de deformidades e contraturas que afetam a funcionalidade, com isso, para minimizar esses efeitos são necessárias medidas preventivas. OBJETIVOS: Desenvolver um aplicativo de orientação familiar para o manejo de pacientes referente a posicionamento, transferências posturais e prevenção de contraturas. MATERIAIS E MÉTODOS: O estudo constituiu-se de uma pesquisa de produção tecnológica, visando o desenvolvimento de um aplicativo móvel para a plataforma Android. A produção do aplicativo dividiu-se em três etapas, pesquisa científica, através das bases de dados de acesso eletrônico como PUBMED, LILACS, MEDLINE e Scielo; elaboração de uma linguagem simplificada e desenvolvimento da aplicação Android. RESULTADOS: O resultado obtido foi um aplicativo móvel composto por 5 tópicos: informações gerais da ECNPI, alongamentos, transferências posturais, manuseio e referências bibliográficas CONCLUSÃO: Verificouse a importância de adequar a assistência em saúde aos avanços tecnológicos para melhoria da qualidade de vida dos pacientes.

PALAVRAS-CHAVE: Paralisia cerebral. Aplicativos móveis. Serviços preventivos de saúde.
ABSTRACT | INTRODUCTION: Non-Progressive Chronic Childhood Encephalopathy (ECNPI) is a condition of non-progressive physical disability due to a developing central nervous system injury. It has motor disorders, muscle tone and posture, thus causing a deficiency in coordination of muscle action and movements, inability or difficulty in maintaining posture. With the growth and development of children may occur an aggravation of health, as the appearance of deformities and contractures that affect functionality, so to minimize these effects preventive measures are necessary. OBJECTIVES: To develop a family guidance application for patient management regarding positioning, transfers and prevention of contractures. MATERIALS AND METHODS: The study consisted of a technological production research, aiming at the development of a mobile application for the Android platform. The production of the application was divided into three stages, scientific research, through electronic access databases such as PUBMED, LILACS, MEDLINE and Scielo; elaboration of a simplified language and development of android application. RESULTS: The result was a mobile application consisting of 5 topics: general ECNPI information, stretching, postural transfers, handling and bibliographic references. CONCLUSION: The importance of adapting health care to technological advances to improve patients' quality of life was verified.

KEYWORDS: Cerebral palsy. Mobile applications. Preventive health services. 


\section{Introdução}

A Encefalopatia Crônica Não Progressiva da Infância (ECNPI) também conhecida como Paralisia Cerebral (PC) é uma condição de deficiência física não progressiva decorrente de uma lesão no sistema nervoso central em desenvolvimento, a qual pode ocorrer no período pré, peri e pós natal. Apresenta desordens motoras, no tônus muscular e na postura, com isso provocando uma deficiência na coordenação da ação muscular e dos movimentos, incapacidade ou dificuldade na manutenção postural' ${ }^{1}$.

As manifestações clínicas motoras são diversas que variam de acordo com seu grau, tipo de acometimento motor e desordem do movimento. Dessa forma, ela é classificada em espástica, atetósica, atáxica, hipotônica e mista e quanto à localização corporal é classificada em hemiparética, diparética e quadriparética. A gravidade do acometimento neuromotor de indivíduos com ECNPI pode ser caracterizada como leve, moderada ou grave, baseada no meio de locomoção².

Com o crescimento e desenvolvimento das crianças pode ocorrer um agravo do quadro de saúde devido às complicações como aparecimento de atrofias, deformidades, alteração do tônus muscular e contraturas. Associado a isso, outros fatores podem interferir e influenciar diretamente no desempenho funcional, como o ambiente e a ausência de estimulação pelos cuidadores pode fazer com que os indivíduos não adquiram maior funcionalidade e sua independência³.

Esses agravos e fatores, dificultam o posicionamento confortável do indivíduo, prejudicam as tarefas de vida diária, posicionamento, transferência e os cuidados de higiene entre outros. Nesse contexto, é de extrema importância a prevenção dessas complicações para oferecer bem-estar e saúde através de orientações aos cuidadores. Sendo assim, essas prescrições fisioterapêuticas são necessárias para integrar e complementar o tratamento realizado em clínica à rotina diária do paciente, visto que, o ambiente domiciliar é o local em que o indivíduo passa a maior parte do tempo ${ }^{4}$.

Atualmente, um meio eficaz de disponibilizar ferramentas, sejam educacionais, de entretenimento, de saúde entre tantas outras, têm sido os softwares para dispositivos móveis, os chamados aplicativos. Essas ferramentas demonstram facilidade de alcançar o público-alvo, devido a rápida disseminação dos dispositivos móveis no mercado a qual tem favorecido a inserção dessa tecnologia na área da saúde, tanto nas pesquisas quanto na assistência e gerenciamento do cuidado. Os aplicativos utilizados na área de saúde apresentam como vantagens a redução de custos com impressões em papéis, a divulgação do material sem necessitar de deslocamento e o alcance de locais longínquos ${ }^{5,6}$.

Dessa forma, associado aos benefícios e à necessidade de orientar cuidadores de crianças com ECNPI, o objetivo deste estudo foi desenvolver um aplicativo móvel que contenha informações e orientações sobre a patologia, transferências posturais, prevenção de contraturas e deformidades em indivíduos com ECNPI.

\section{Materiais e métodos}

O estudo constitui-se de uma pesquisa de produção tecnológica, caracterizada por tratar-se do processo de desenvolvimento e criação de um aplicativo móvel com orientações domiciliares para cuidadores de indivíduos com ECNPI quanto às transferências posturais, posicionamento, prevenção de contraturas e deformidades.

A primeira etapa do desenvolvimento do aplicativo móvel consistiu na pesquisa científica sobre ECNPI e patologias associadas, deformidades, contraturas, sobre posicionamentos e transferências posturais adequadas para fundamentação do tema proposto e estabelecimento de um princípio norteador para indicar as principais demandas do público-alvo. Desta forma, foram utilizadas as bases de dados de acesso eletrônico PUBMED, LILACS, MEDLINE e Scielo utilizando os seguintes descritores do DECS: (Descritores em Ciência da Saúde) Paralisia cerebral (Cerebral Palsy), Educação (Education), Telemedicina (Telemedicine) e Cuidadores (Caregivers) de forma isolada e associada.

Foram pesquisados artigos originais, dissertações e revisões de literatura sistemática, integrativa ou narrativa disponíveis na íntegra, com período de publicação específico de 2015 a 2019, nos idiomas inglês e português. Foram excluídos artigos com duplicidade, estudos editoriais, anais de congresso, estudos de casos e artigos de reflexão. A busca aconteceu no período de setembro a novembro de 2018. 
Na segunda etapa foram realizadas, por uma fisioterapeuta especialista em reabilitação neurológica, correções na terminologia utilizada conforme a característica do público-alvo, visando torná-la acessível. Na terceira etapa ocorreu o desenvolvimento do aplicativo, onde foram utilizadas ferramentas para desenvolvimento de sistema para plataforma Android, ou seja, bibliotecas e ferramentas na linguagem de programação Java fornecida pelo Android Kit de Desenvolvimento de Software (SDK). Pois a criação da aplicação só é possível após a preparação do ambiente de trabalho que ocorre com a instalação e configuração do Android SDK (contendo SDK Tools, Build Tools e o Platform Tools) e da IDE (Integrated Development Environment) de programação Android Studio.

\section{Resultados}

Para fundamentação teórica do tema, foram revisados diversos estudos. Os autores mais relevantes, para presente pesquisa, são evidenciados no Quadro 1.

Quadro 1. Estudos de base para a fundamentação teórica do aplicativo

\begin{tabular}{|c|c|c|}
\hline AUTORES & TITUULO DO ESTUDO & RESULTADOS \\
\hline $\begin{array}{l}\text { Santos GFL, } \\
\text { Santos FF, } \\
\text { Martins } \\
\text { FPAA }\end{array}$ & $\begin{array}{l}\text { Atuação da fisioterapia na } \\
\text { estimulação precoce em } \\
\text { criança com paralisia } \\
\text { cerebral. }\end{array}$ & $\begin{array}{l}\text { Evidenciou-se que técnicas fisioterapêuticas contribuem } \\
\text { para o aprendizado motor, devendo ser realizadas nos } \\
\text { primeiros anos de vida, no auge da neuroplasticidade } \\
\text { para atingir resultados mais significativos, obtendo, } \\
\text { assim, um efeito mais próximo possível do } \\
\text { desenvolvimento típico. }\end{array}$ \\
\hline $\begin{array}{l}\text { Alflen RA, } \\
\text { Lima LD, } \\
\text { Bussador A, } \\
\text { Peres LW, } \\
\text { Junior JA }\end{array}$ & $\begin{array}{l}\text { Desenvolvimento de uma } \\
\text { plataforma para auxílio na } \\
\text { fisioterapia de pacientes } \\
\text { com encefalopatia crônica } \\
\text { não progressiva da } \\
\text { infância - ECNPI }\end{array}$ & $\begin{array}{l}\text { Presente trabalho apresentou o protótipo desenvolvido } \\
\text { em uma parceria dos cursos de Ciência da Computação } \\
\text { e Fisioterapia para auxílio no tratamento de crianças } \\
\text { com paralisia cerebral, embutindo nas sessốes } \\
\text { conceitos de gameterapia. }\end{array}$ \\
\hline Barbosa JM & $\begin{array}{l}\text { Orientações sobre } \\
\text { cuidados de crianças com } \\
\text { paralisia cerebral para } \\
\text { cuidadores e profissionais } \\
\text { da saúde: um manual } \\
\text { prático. }\end{array}$ & $\begin{array}{l}\text { Este manual foi composto por duas partes: a primeira } \\
\text { que aborda o significado, tipos, causas e consequências } \\
\text { da PC e a segunda que apresenta sugestôes para } \\
\text { facilitar a alimentação, vestuário, higiene, banho, } \\
\text { somadas às orientaçóes de posicionamento, exercícios } \\
\text { de alongamentos e brincadeiras. }\end{array}$ \\
\hline $\begin{array}{l}\text { Souza JS, } \\
\text { Knobel KAB. }\end{array}$ & $\begin{array}{l}\text { Guia ilustrado de } \\
\text { orientações a cuidadores } \\
\text { de crianças com } \\
\text { deficiências } \\
\text { neuromotoras. }\end{array}$ & $\begin{array}{l}\text { O presente guia ilustrado contém informações } \\
\text { referentes aos cuidados e manejos de crianças com } \\
\text { deficiência neuromotora. Ele foi feito para incentivar o } \\
\text { leitor a estimular o desenvolvimento neuropsicomotor } \\
\text { de crianças com deficiência neuromotora. }\end{array}$ \\
\hline $\begin{array}{l}\text { Pavão SL, } \\
\text { Silva FPS, } \\
\text { Rocha NAC. }\end{array}$ & $\begin{array}{l}\text { Efeito da orientação } \\
\text { domiciliar no } \\
\text { desempenho funcional de } \\
\text { crianças com } \\
\text { necessidades especiais. }\end{array}$ & $\begin{array}{l}\text { Sugere-se, por meio dos resultados, que a orientação } \\
\text { domiciliar e individualizada a cuidadores seja eficaz } \\
\text { para elevar o nivel de funcionalidade de crianças com } \\
\text { necessidades especiais em áreas de autocuidado e } \\
\text { função social de acordo com o PEDI. Sendo, assim, } \\
\text { uma forma simples e eficiente de intervenção junto à } \\
\text { família. }\end{array}$ \\
\hline
\end{tabular}


O aplicativo foi intitulado Mova+ e foi desenvolvido para aparelhos que operam por tecnologia do tipo Android, a escolha por esta compatibilidade deveu-se ao fato de que este é o tipo de sistema operacional compatível com a maioria dos aparelhos celulares, o que torna o aplicativo acessível a um maior número de pessoas.

O produto funciona como uma cartilha digital e à medida que a instalação do aplicativo for concluída ficará disponível também para uso off-line, sem a necessidade de acesso à internet para utilizá-lo. Quanto à linguagem utilizada primou-se pela simplicidade e objetividade, tornando os conteúdos mais acessíveis ao público-alvo. Sobre o design priorizou-se design leve e limpo, com plano de fundo em cores claras.

Os dados foram organizados no conceito estrutural de árvore para serem acessados internamente, os menus estão mais próximos da raiz (menu principal) e páginas contendo as informações estão nas folhas, tendo assim uma forma mais organizada de trabalhar com os objetos e exibir as informações. A Figura 1 representa a estrutura em árvore trabalhada no projeto.

Figura 1. Estrutura de árvore

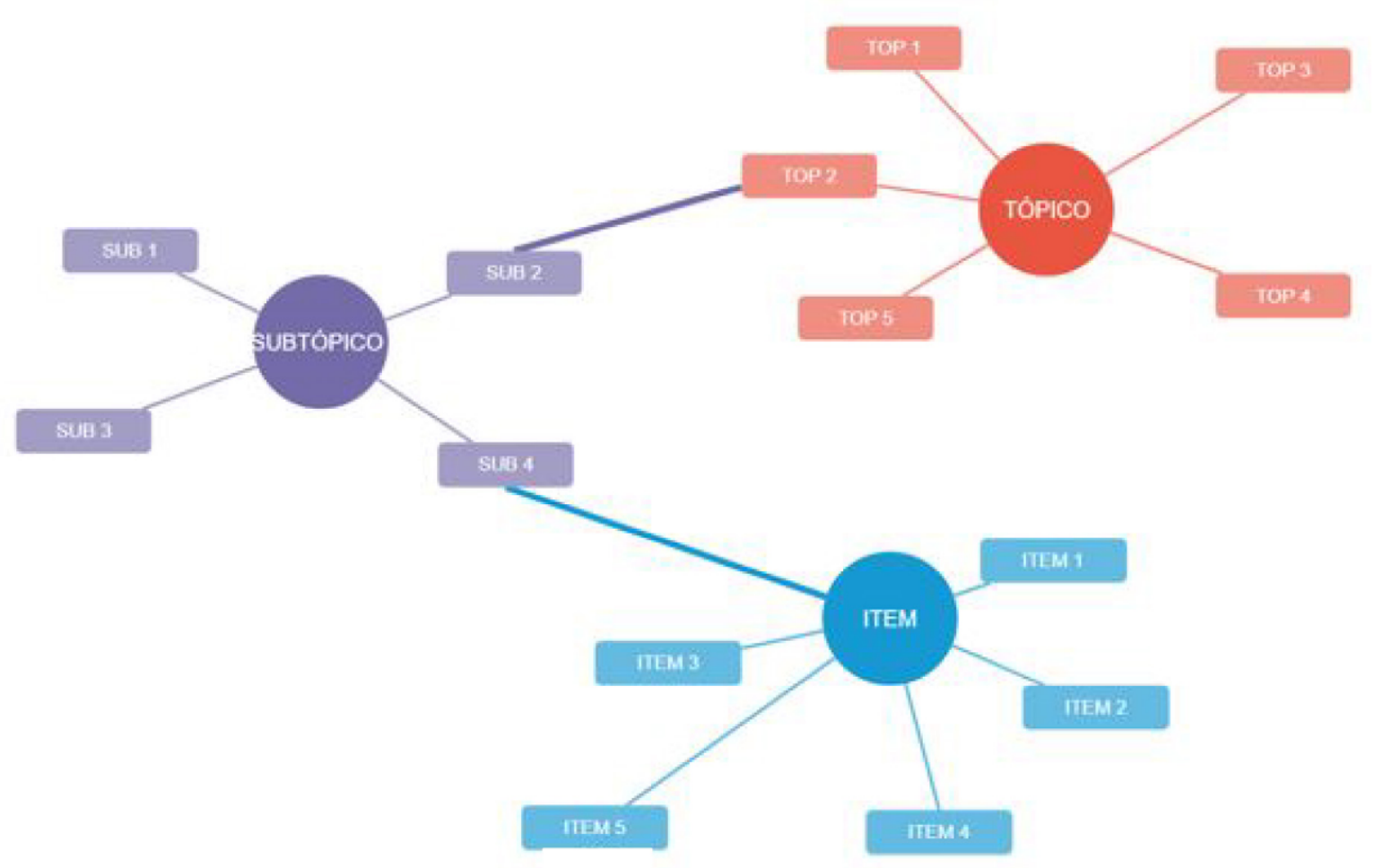

Sendo assim, a aplicação possui somente 3 telas que são ajustadas em tempo de execução, fazendo com que o tamanho do aplicativo seja reduzido ao evitar o excesso de telas. Todos os dados são inseridos em um HashMap usado para consultas mais eficientes do que em comparação com Listas e Arrays, pois funciona com a implementação de chave-valor através de um ID para cada objeto tratado. A Figura 2 demonstra como ocorre a troca de informações por meio do mapeamento por ID. 


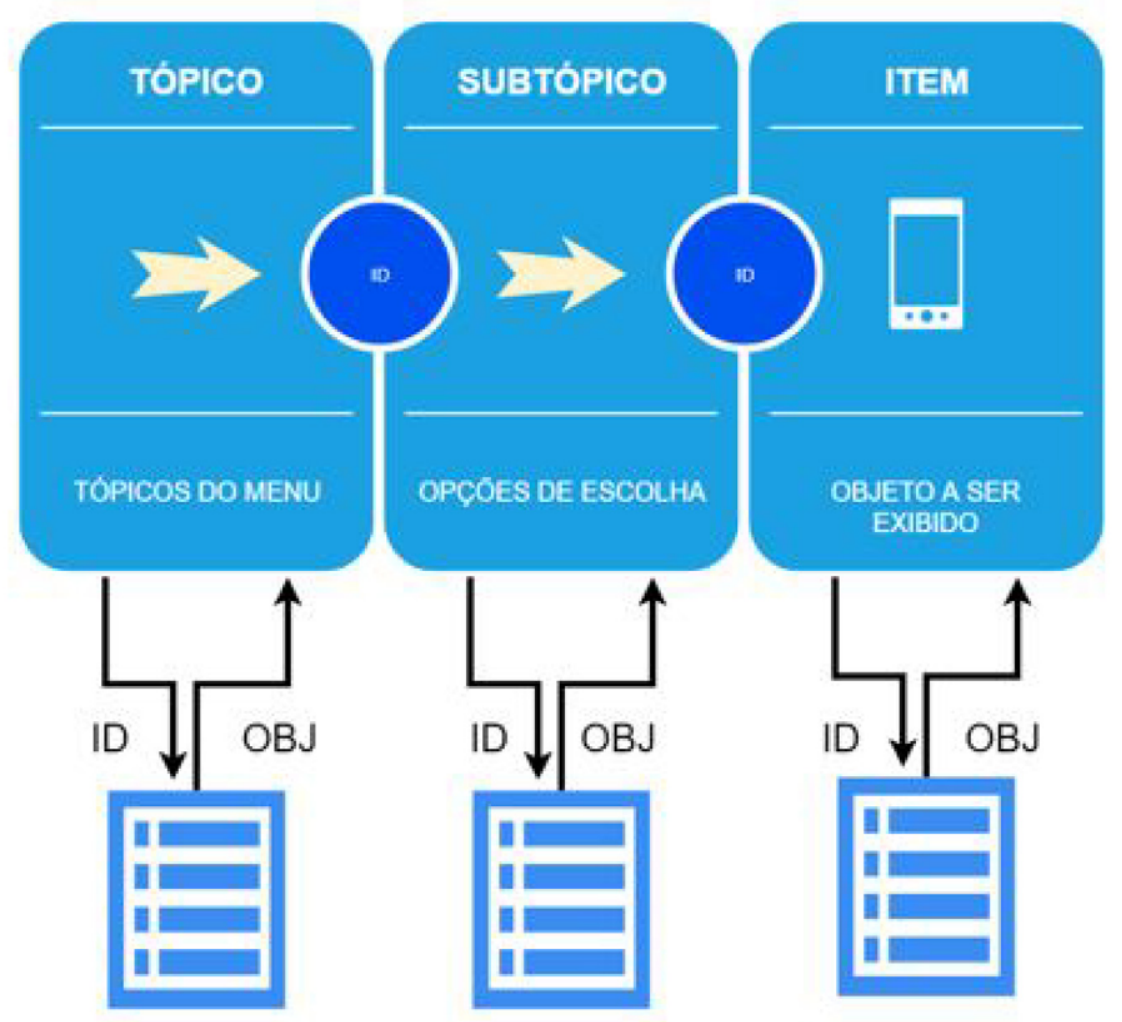

Cada item do HashMap é composto por uma chave e um objeto como o valor, o objeto possui todas as informações da tela que será exibida, como texto e imagens. Essa forma de implementação é característica de sistemas escaláveis, pois uma vez que implementado a atualização ocorre com a inserção ou remoção de um objeto, não sendo necessário criar ou remover novas telas.

O produto é composto por 6 tópicos, o primeiro tópico aborda sobre informações gerais como a definição da ECNPI, espasticidade, dicas sobre profissionais que podem auxiliar no tratamento dos indivíduos e esclarecimento sobre a utilização de órteses. Em seguida os demais tópicos são: Manuseio, Posturas, Alongamentos, Referências Bibliográficas utilizadas para a elaboração do produto e Sua Opinião para os usuários avaliarem o aplicativo. A figura 3 mostra a tela inicial com todas as possibilidades de acesso no sistema. 


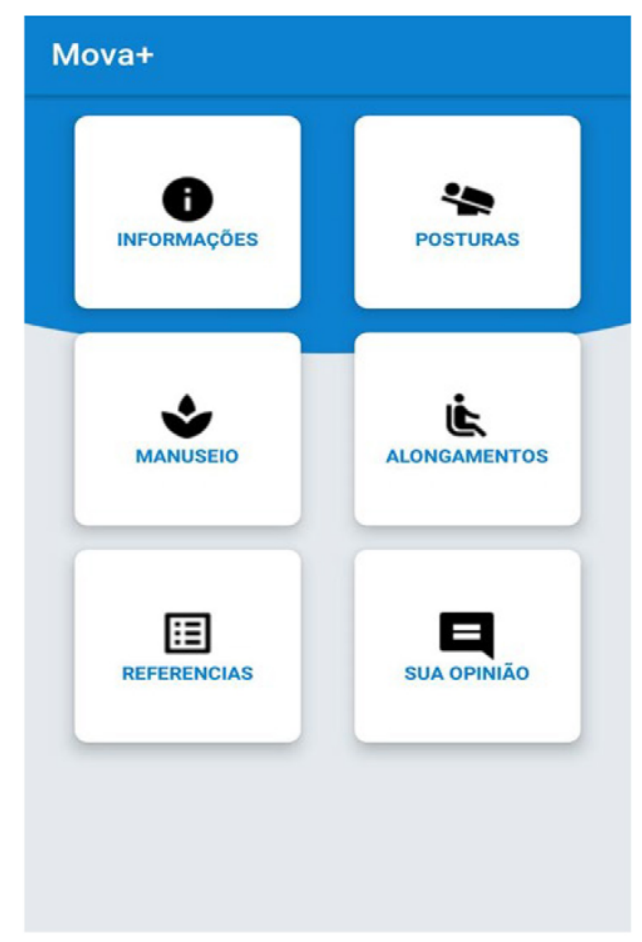

Cada tópico contém textos explicativos e imagens ilustrativas. Na figura 4 observa-se os principais grupos musculares que foram escolhidos para os alongamentos e a demonstração de como realizar o alongamento com a finalidade de prevenir e reduzir contraturas e deformidades.

Figura 4. Tópico do aplicativo referente aos alongamentos. Imagem de alongamento ilustrativa autoral

\begin{tabular}{l} 
ALONGAMENTO \\
Músculos Flexores do Quadril \\
Músculos Adutores do Quadril \\
Músculos Flexores do Joelho \\
Músculos Flexores do Tornozelo \\
Músculos Adutores do Ombro \\
Músculos Rotadores do Ombro \\
\hline Músculos Flexores do Cotovelo \\
\hline Músculos Flexores do Punho
\end{tabular}

\section{Movat}

A pessoa que estar de barriga para baixo e o cuidador ao lado. A mâo do cuidador fica no bumbum para impedir que ela se movimente e a outra mão acima do joelho de forma que a perna seja elevada.

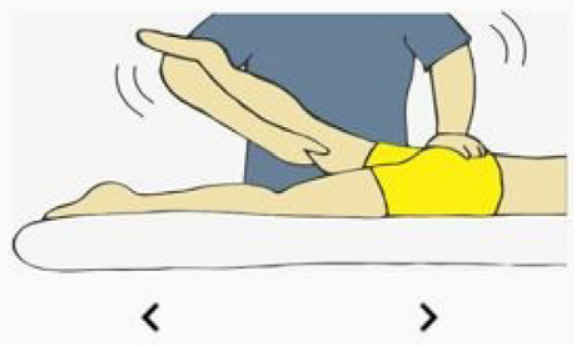

Na figura 5, o tópico manuseio demonstra a forma correta para manipulação da criança, foram escolhidas as abordagens que são mais suscetíveis a lesões se não forem feitas de maneira adequada. 
Figura 5. Tópico do aplicativo referente ao manuseio e exemplo ilustrado autoral do manuseio da cabeça

\section{MANUSEIO}

Pé Equino

\section{Cabeça para Trás}

Pernas em Tesoura(Cruzadas)

Braços

\section{Movat}

Năo se deve corrigir essa postura colocando as măos atrás da cabeça, pois fará que a pessoa jogue a cabeça ainda mais para trás. A forma mais adequada é colocar as măos em cada lado da cabeça e puxar para cima.

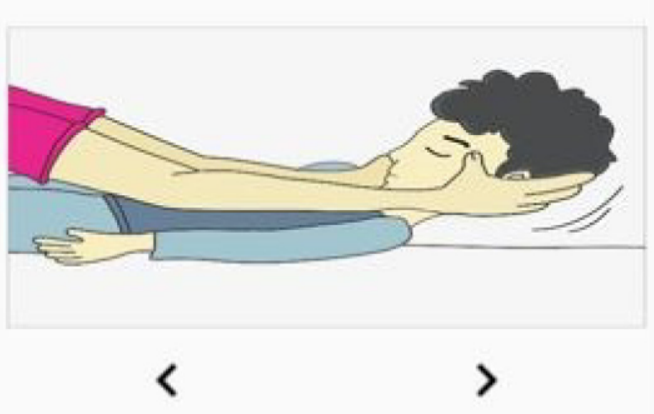

No tópico Postura, observado na figura 6 são abordadas nas abas as orientações gerais e a forma adequada de posicionar as crianças de decúbito dorsal e ventral, carregar no colo, sentado, em pé e os cuidados com as lesões por pressão.

Figura 6. Tópico referente sobre postura adequada. Imagem ilustrativa autoral

\section{Mova+}

o pescoço, o adulto pode sentar a criança no seu colo,

apoiando o pescoço a cabeça da criança uma das

mãos e segurando o tronco da criança com a outra

Além de estimular os músculos da postura.

DICAS

- As crianças ou adolescentes que apresentam alguma dificuldade para sentar deve-se colocar um apoio para as costas travesseiros ou almofadas firmes, o qual também, evita que eles caiam para os lados.

- Sentar a criança ou adolescente no centro de uma bóia inflável ou de uma câmara de pneu.

- Posicionar a criança ou adolescente no sofá, na quina entre o braço e o encosto.

- A forma correta para sentar no chão é com as pernas esticadas para evitar que a criança cruze as pernas ou adote a postura em $\mathrm{W}$

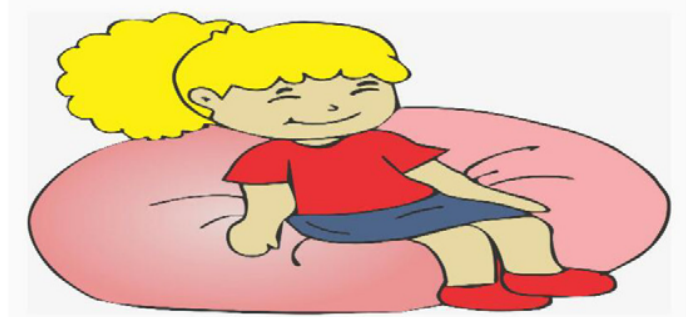


O tópico 5 contêm Referências Bibliográficas utilizadas para a produção do Mova+, sobre as quais os usuários têm a liberdade para consultá-las caso necessário. Isto evita que os mesmos consultem informações adicionais de fontes desconhecidas e sem comprovação científica.

O tópico 6 , destinado à opinião dos usuários foi desenvolvido para tornar viável ao público relatar se o aplicativo Ihes foi benéfico, oferecer sugestões de melhoria, além de realizar perguntas pertinentes a temática. Este espaço destinado ao feedback sobre o aplicativo caracteriza-se como de grande importância para realizar atualizações e melhorias.

\section{Discussão}

O aplicativo desenvolvido proporciona aos cuidadores de crianças com ECNPI instruções adequadas para o cuidado domiciliar para a maximização dos efeitos do tratamento realizado em ambulatório fisioterapêutico ${ }^{7}$. Ele apresenta aos usuários possibilidade de consultas sempre que lhes surgir alguma dúvida sobre os assuntos abordados, uma vez que ele oferece a possibilidade de ser consultado em dispositivos móveis e na modalidade off-line ${ }^{8}$.

Uma das principais vantagens do desenvolvimento de aplicações móveis educacionais é o reaproveitamento, pois, por meio da disponibilidade na internet permitem o acesso e a consulta dessas informações em qualquer tempo, lugar e quantas vezes forem necessárias 9 .

Considerando que um dos objetivos de um aplicativo é facilitar a realização de uma tarefa específica ou auxiliar em algum serviço, ao tratar-se das ciências da saúde pode-se afirmar que a disponibilização de conhecimento científico à um clique de distância, amplia a capacidade de empoderamento do paciente e de seus cuidadores diante da sua realidade ${ }^{10}$. Por este motivo, o aplicativo desenvolvido obteve fundamentação teórica e prática em bancos de dados confiáveis e seguros, além de transmitir as orientações aos cuidadores através de uma linguagem apropriada e simplificada ${ }^{11}$.

Por muitas vezes a família não é orientada pelos serviços de saúde sobre como realizar o cuidado no ambiente domiciliar, o que aponta que ela não é re- conhecida como principal unidade de cuidado. Isto implica em maiores riscos funcionais para o paciente frente ao manejo inadequado e a escassez de estímulos. A produção de aplicativos educativos na área da saúde ameniza esta falha no serviço de saúde e prepara a família para o cuidado em domicílio ${ }^{12}$.

Orientação aos cuidadores de crianças com necessidades especiais, quanto ao cuidado domiciliar, apresenta efeito direto no aumento do desempenho funcional da criança nas atividades de vida diárias, nas áreas de autocuidado, mobilidade e função social. O aplicativo desenvolvido pode ampliar a relação da criança com o ambiente em que ela se encontra, tornando o local um agente facilitador da funcionalidade, ao invés de um fator limitante. Deste modo as orientações domiciliares ampliam o processo terapêutico para além do atendimento ambulatoria| ${ }^{13,14}$.

É escasso o conhecimento sobre o entendimento da doença por parte dos cuidadores, assim como sobre os aspectos relacionados aos cuidados diários domiciliares prestados à criança com ECNPI. A dificuldade dos cuidadores em lidar com as limitações da criança pode promover situações resultantes em desgaste físico e emocional significativos para o mesmo. O uso de um aplicativo com orientações para manejo de posicionamento, transferências posturais e prevenção de contraturas pode beneficiar o processo de reabilitação da criança e ainda proporcionar diminuição das sobrecargas, redução das demandas físicas e a prevenção de lesões por esforços repetitivos inadequados dos cuidadores ${ }^{15,16}$.

No estudo de Ghazisaeedi et al. (2016), foram avaliados os efeitos de uma aplicação Android sobre o conhecimento de cuidadores de crianças com ECNPI antes e após 2 meses da utilização da aplicação. Este aplicativo incluía informações educativas sobre alimentação, banho, brincar, manejo e movimentos. Como desfecho, os mesmos obtiveram resultados que corroboram com o aumento do conhecimento dos cuidadores em todos os domínios, exceto o brincar ${ }^{17}$.

Neste sentido, destaca-se um resultado positivo da utilização das tecnologias para incrementar o conhecimento dos cuidadores de crianças com ECNPI acerca da realização adequadas das atividades diárias.

Embora existam relatos do desenvolvimento de outros aplicativos móveis aplicáveis a pacientes com ECNPI 17,18,19, trata-se de um campo ainda reduzido, 
mas com grande potencial e em crescimento que tem chamado a atenção de pesquisadores que reforçam o papel fundamental da pesquisa sobre o uso dos aplicativos em saúde ${ }^{20}$.

Tem-se como limitações do estudo o desenvolvimento do aplicativo na plataforma Android, que, por mais que abranja um maior número de possíveis usuários, o ideal é que tal tecnologia seja adequada também a outros sistemas operacionais. Pontua-se a necessidade de finalização da fase de teste e verificação do impacto do aplicativo sobre o público-alvo, oferecer melhorias e por fim, realizar a implementação do aplicativo, sendo esta uma proposta para estudos posteriores.

\section{Conclusão}

Este estudo foi um registro sobre a criação de uma nova tecnologia, o aplicativo "Movat" destinado a cuidadores de indivíduos com ECNPI, o qual aborda sobre como realizar transferências posturais, posicionamento, prevenção de contraturas e deformidades, utilizando a plataforma Android. Vale ressaltar que ainda se faz necessária a realização de testes para verificar a necessidade de novos ajustes e atualizações.

Portanto, espera-se com essa pesquisa despertar o interesse de outros pesquisadores em saúde para o desenvolvimento de novas tecnologias alinhando o conhecimento teórico-prático da fisioterapia e buscando a melhoria da qualidade da assistência às pessoas com a ECNPI e qualidade de vida dos cuidadores e dos pacientes.

\section{Contribuições dos autores}

Dias ACL, Paz COC e Rocha LSO participaram da concepção, delineamento, busca e análise dos dados da pesquisa, interpretação dos resultados e redação do artigo científico. Carvalho IS participou da concepção e do desenho do estudo, elaboração do produto, interpretação dos dados e redação do artigo científico. Gomes IF participou da coleta e da análise dos dados da pesquisa, interpretação dos resultados, redação do artigo científico. Costa CA e Moraes JBA participaram da análise dos dados, concepção do estudo e redação do artigo científico.

\section{Conflitos de interesses}

Nenhum conflito financeiro, legal ou político envolvendo terceiros (governo, empresas e fundações privadas, etc.) foi declarado para nenhum aspecto do trabalho submetido (incluindo mas não limitando-se a subvenções e financiamentos, participação em conselho consultivo, desenho de estudo, preparação de manuscrito, análise estatística, etc.).

\section{Referências}

1. Santos GFL, Santos FF, Martins FPAA. Atuação de Fisioterapia na Estimulação Precoce em Criança com Paralisia Cerebral. DêCiência em Foco. 2017;1(2):76-94.

2. Alflen RA, Lima LD, Bussador A, Peres LW, Aikes Junior J. Desenvolvimento de uma plataforma para auxílio na fisioterapia de pacientes com encefalopatia crônica não progressiva da infância-ECNPI. Rev Eletrônica Científica Inovação e Tecnologia. 2016;1(13):28-37.

3. Barbosa JM. Orientações sobre cuidados de crianças com paralisia cerebral para cuidadores e profissionais da saúde: um manual prático. [Dissertação de mestrado]. Minas Gerais: Universidade Federal de Viçosa; 2015.

4. Toledo CAW, Pereira CHCN, Vinhaes MM, Lopes MIR, Nogueira MARJ. Perfil epidemiológico de crianças diagnosticadas com paralisia cerebral atendidas no Centro de Reabilitação Lucy Montoro de São José dos Campos. Acta Fisiatr. 2015;22(3):118122. doi: $\underline{10.5935 / 0104-7795.20150023}$

5. Tibes CMS. Aplicativo móvel para prevenção e classificação de úlceras por pressão. [Dissertação de Mestrado]. São Carlos: Universidade Federal de São Carlos; 2015.

6. Vêscovi SJB, Primo BB, Sant' Anna HC, Briguete MEO, Rohr RV, Prado TN et al. Aplicativo móvel para avaliação dos pés de pessoas com diabetes mellitus. Acta Paul Enferm. 2017;30(6):60713. doi: $\underline{10.1590 / 1982-0194201700087}$

7. Rocha TAH, Fachini L A., Thumé E, Silva NC, Barbosa ACQ, Carmo M. Saúde Móvel: novas perspectivas para a oferta de serviços em saúde. Epidemiol. Serv. Saúde. 2016; 25(1):159-170. doi: $10.5123 / S 1679-49742016000100016$

8. Kao CK, Liebovitz DM. Consumer mobile health apps: current state, barriers, and future directions. PM\&R. 2017; 9(5): 106-115. doi: 10.1016/j.pmrj.2017.02.018

9. Pereira FGF, Silva DV, Sousa LMO, Frota NM. Construção de um aplicativo digital para o ensino de sinais vitais. Rev. Gaúcha Enferm. 2016;37(2). doi: 10.1590/1983-1447.2016.02.59015 
10. Rodríguez MTS, Vázquez SC, Casas PM, Cuerda RC. Apps en neurorrehabilitación. Una revisión sistemática de aplicaciones móviles. Neurología. 2018; 33(5):313-326. doi: 10.1016/j. $\underline{\text { nrl.2015.10.005 }}$

11.Souza JS, Knobel KAB. Guia ilustrado de orientações a cuidadores de crianças com deficiências neuromotoras. ConScientiae Saúde. 2019;18(1):8-17. doi: 10.5585/ConsSaude. v18n1.8617

12. Dias BC et al. Crianças com Cuidados Complexos no Domicílio. Esc Anna Nery 2019;23(1):e20180127. doi: 10.1590/2177-9465-

EAN-2018-0127

13. Pavão SL, Silva FPS, Rocha NAC. Efeito da orientação domiciliar no desempenho funcional de crianças com necessidades especiais. Motricidade. 2011;7(1):21-9.

14. Liboni LS, Leite E, Sacramento FCR, Oliveira RFR, Correa CF. Impacto Social de uma Criança com Paralisia Cerebral na Vida de um Cuidador Familiar. R. Enferm. UFJF. 2018; 4(1): 39-50. doi: 10.34019/2446-5739.2018.v4.14015

15. Santos AAS, Oliveira CCC, Vargas MM, Macedo IAB. Avaliação da sobrecarga dos cuidadores de crianças com paralisia cerebral. Ciência, Cuidado e Saúde. 2015; 9(3):503-509. doi: 10.4025/ cienccuidsaude.v9i3.9405

16. Oliveira FL, Dounis AB. As alterações na dinâmica familiar diante do diagnóstico da criança com Paralisia Cerebral: Estado da Arte. Revista de Psicologia. 2012; 3(1): 18-27.

16. Ghazisaeedi M, Safari A, Sheikhtaheri A, Dalvand H. The effect of an android-based application on the knowledge of the caregivers of children with cerebral palsy. Med J Islam Repub Iran. 2016; 30:456.

17. Mariblanca MR, Cuerda RC. Aplicaciones móviles en la parálisis cerebral infantil. Neurología. 2017. doi: 10.1016/j.nrl.2017.09.018

18. Lima JJ, Vieira LGD, Nunes MM. Computerized nursing process: development of a mobile technology for use with neonates. Rev Bras Enferm. 2018;71(Suppl 3):1273-80. doi: 10.1590/0034-71672017-0267

19. Spittle AJ, Olsen J, Kwong A, Doyle LW, Marschik PB, Einspieler $C$ et al. The Baby Moves Prospective Cohort Study Protocol: Using a Smartphone Application With The General Movements Assessment to Predict Neurodevelopmental Outcomes at Age 2 Years for Extremely Preterm or Extremely Low Birthweight Infants. pen do BMJ de 2016;6(10):e013446. doi: 10.1136/ bmjopen-2016-013446

20. Khanna M, Gowda GS, Bagevadi VI, Gupta A, Kulkarni K, S Shyam RP et al. Feasibility and Utility of Tele-Neurorehabilitation Service in India: Experience from a Quaternary Center. J Practo Rural Neurosci. 2018;9(4):541-544. doi: 10.4103/jnrp.jnrp_104_18 